\title{
From Business Intelligence to Artificial Intelligence
}

\author{
Bahman Zohuri ${ }^{1 *}$ and Masoud Moghaddam ${ }^{2}$ \\ ${ }^{1}$ Electrical and Computer Engineering Department, University of New Mexico, Albuquerque, New Mexico, USA \\ ${ }^{2}$ Galaxy Advance Engineering Consultant, Albuquerque, New Mexico, USA
}

*Corresponding author: Bahman Zohuri, Electrical Engineering and Computer Science Department, Albuquerque, and Galaxy Advanced Engineering (CEO), New Mexico, USA

\begin{abstract}
With today's growing information and overloading of its volume based on tremendous size of data growing to the level of big data, Business Intelligence (BI) is not enough to handle any day-to-day business operation of any enterprises. It is becoming tremendously difficult to analyze the huge amounts of data that contain the information and makes it very strenuous and inconvenient to introduce an appropriate methodology of decision-making fast enough to the point that it can be, considered as real time, a methodology that we used to call it BI. The demand for real time processing information and related data both structured and unstructured is on the rise and consequently makes it harder and harder to implement correct decision making at enterprise level that was driven by $\mathrm{BI}$, in order to keep the organization robust and resilient against either man made threats or natural disasters. With smart malware in modern computation world and necessity for Internet-of-Things (IoT), we are in need of a better intelligence system that today we know it as Artificial Intelligence (AI). AI with its two other subset that are called Machine Learning (ML) and Deep Learning (DL), we have a better chance against any cyber-attack and makes our day-to-day operation within our organization a more robust one as well makes our decision making as stakeholder more trust worthy one as well.
\end{abstract}

Keywords: Resilience System; Business Intelligence; Artificial Intelligence; Cyber Security; Decision Making in Real Time; Machine Learning; Deep Learning

\section{Introduction}

Today's campaign against any cyber-attack has put a huge demand on cyber security and on information security folks at different levels of any organization. Therefore, processing incoming data as sets of information becomes more and more critical. Furthermore, the data are often, imprecise and will include both quantitative and qualitative elements. For these reasons it is important to extend traditional decision-making processes by adding intuitive reasoning, human subjectivity and imprecision. To enhance this process of decision-making, these authors have taken an unorthodox approach by applying a new growing technology known as neural network as part of driving infrastructure for artificial intelligence system to take over from human being in order to satisfy the demand for real time decision making. As far as Business Intelligence (BI) is concerns, the term Business Intelligence refers to technologies, applications and practices for the collection, integration, analysis, and presentation of business information across the enterprises and organizations. The purpose of Business Intelligence is to support better business decision making. Essentially, Business Intelligence systems are data-driven Decision Support Systems (DSS). Business Intelligence is sometimes used interchangeably with briefing books, report and query tools and executive information systems.

\section{What is Business Intelligence (BI)}

Business Intelligence systems provide historical, current, and predictive views of business operations, most often using data that has been gathered into a data warehouse or a data mart and occasionally working from operational data. Software elements support reporting, interactive "slice-and-dice" pivot-table analyses, visualization, and statistical data mining. Applications tackle sales, production, financial, and many other sources of business data for purposes that include business performance management. Information is often gathered about other companies in the same industry which is known as benchmarking and they are competitors in same domain or produced products that are manufactured or presented in the similar marketplace. Currently organizations are starting to see that data and content should not be considered separate aspects of information management, but instead should be managed in an integrated enterprise approach. Enterprise information management brings Business Intelligence and 
Enterprise Content Management together. Currently organizations are moving towards Operational Business Intelligence which is currently under served and uncontested by vendors. Traditionally, Business Intelligence vendors are targeting only top the pyramid but now there is a paradigm shift moving toward taking Business Intelligence to the bottom of the pyramid with a focus of selfservice business intelligence. As we have expressed in some of our published books and articles in various journals "Knowledge is Power in Four Dimensions" 1-2 and order to have the knowledge, one needs Information and Information comes from Data as presented in (Figure 1) here. As Figure 1 indicates the main core of the power, consequently knowledge, information and data rests on the Business Resilience System (BRS) Risk Atom as illustrated in (Figure 2) here.

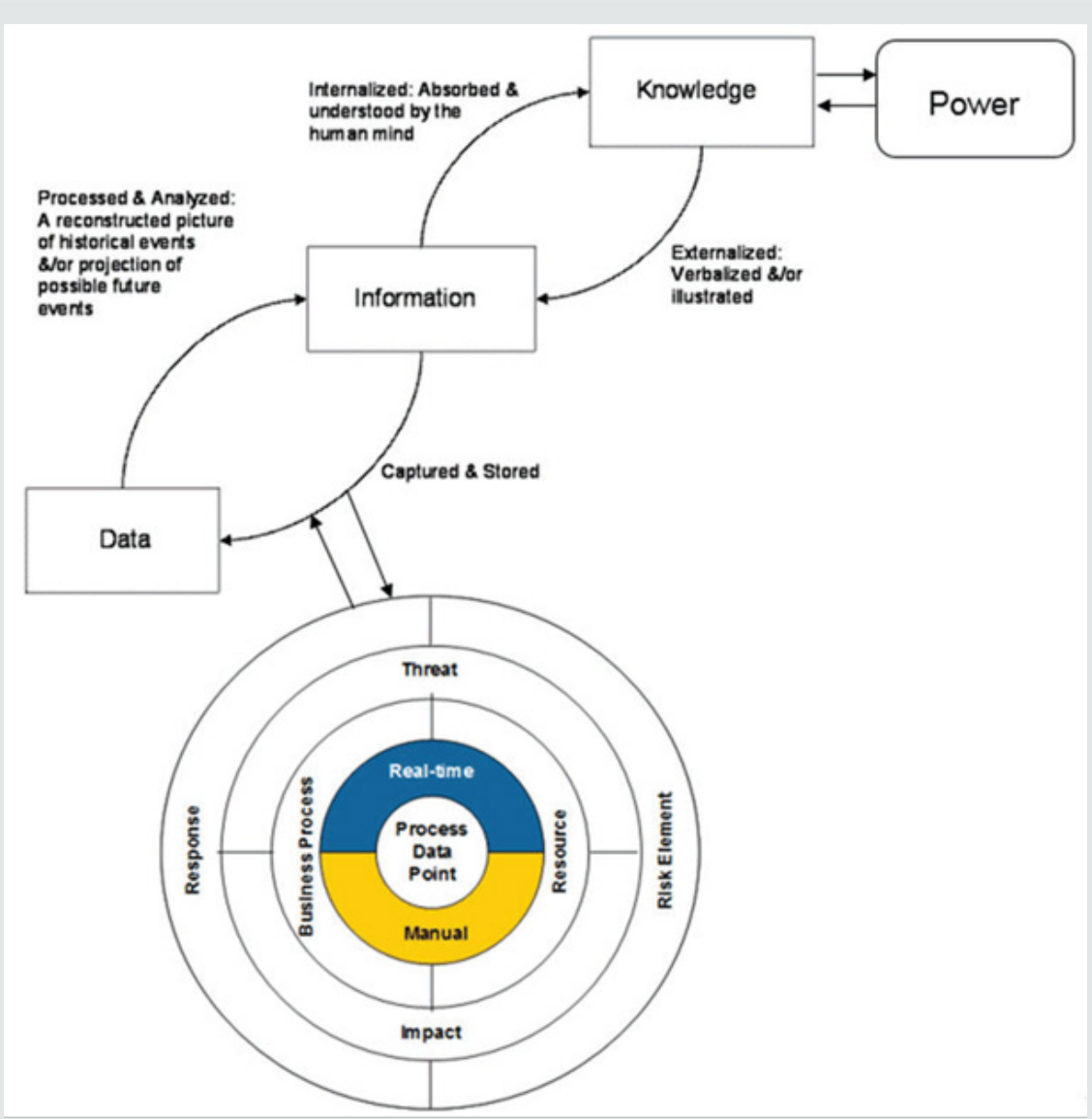

Figure 1: Depiction of Data, Information, and Knowledge is Power in Four Dimensions.

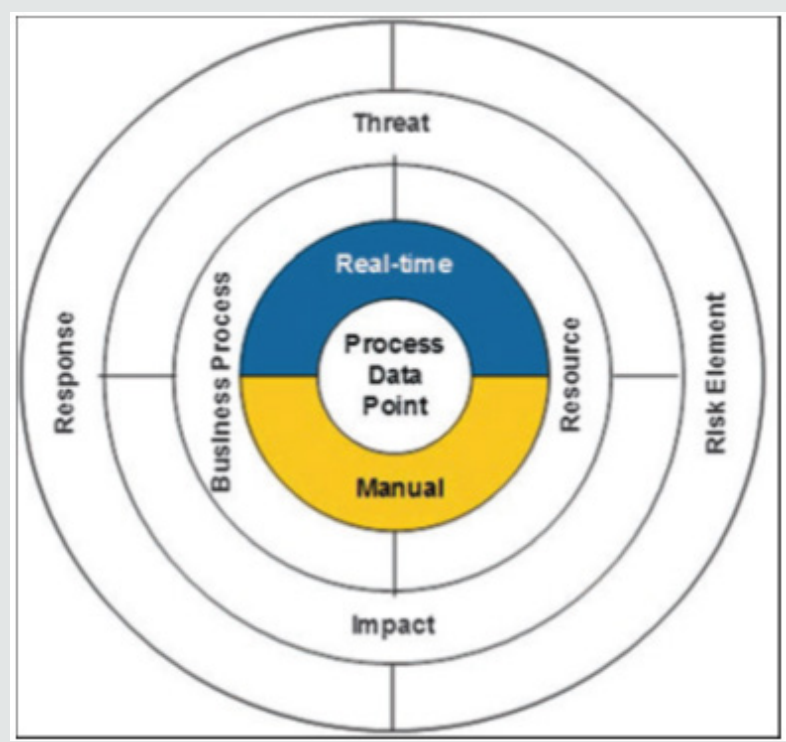

Figure 2: The Business Resilience System Risk Atom. 
Bear in mind that, one of the critical aspects of the Business Resilience System is the Risk Analysis and Risk Assessment Process. However, a critical aspect of the risk analysis process is to identify the preparedness and preventive measures in place at any point in time. Once the potential areas of high exposure to the organization are identified, additional preventative measures can be considered for implementation. Regardless of the prevention techniques employed, possible threats that could arise inside or outside the organization need to be assessed. Although the exact nature of potential disasters or their resulting consequences are difficult to determine, it is beneficial to perform a comprehensive risk assessment of all threats that can realistically occur to the organization. Regardless of the type of threat, the goal of business recovery planning is to ensure the safety of customers, employees, and other personnel during and following a disaster. The relative probability of a disaster occurring should be determined. Items to consider in determining the probability of a specific disaster should include, but not be limited to: Geographic location.

a. Topography of the area.

b. Proximity to major sources of power.

c. Bodies of water and airports.

d. Degree of accessibility to facilities within the organization.

e. History of local utility companies in providing uninterrupted services.

f. History of the area's susceptibility to natural threats.

g. Proximity to major highways, which transport hazardous waste and combustible products.

The above are few natural disasters that one can think of it and we are certain there is more to think about it. However, the human threat is a different ball game that our intelligent communities under the umbrella of Department of Homeland Security need to be involved and their data need to be built in a secure Master Data Management (MDM) or Big Data (BD) system with infrastructure in cloud and strong firewall, which prevents any hacker to penetrate the security of this firewall from cyber-attack perspective. In conclusion, For any critical business process, there may be one or multiple Risk Atoms, but any Risk Atom must reflect a critical business process measure that, when "tipped, "it will begin degrading process capabilities, and, if left unchecked, it will result in a disaster/destruction situation requiring the invocation of a Business Continuity Process (BCP). A PDP can "move" through various levels of thresholds (as a result of threat manifestation) which will determine the type of business activities to be performed to remedy any foreseeable process degradation before it becomes process destruction. Furthermore, as complement to Business Intelligence, we talk about another aspect of it known as SelfService Business Intelligence (SSBI), which involves the business and data analytics that give business end-users access to an organization's information without direct Information Technology (IT) involvement.

Self-service Business intelligence gives end-users the ability to do more with their data without necessarily having technical skills. These solutions are usually created to be flexible and easy-to-use so that end-users can analyze data, make decisions, plan and forecast on their own. Companies such as PARIS Technologies have taken an approach to making Business Intelligence an easily integrated tool for other end-user tools such as Microsoft Excel, Access, Web browsers and other vendors. Bottom line, Business intelligence comprises the strategies and technologies used by enterprises for the data analysis of business information. BI technologies provide historical, current and predictive views of business operations. Business intelligence (BI) leverages software and services to transform data into actionable insights that inform an organization's strategic and tactical business decisions. BI tools access and analyze data sets and present analytical findings in reports, summaries, dashboards, graphs, charts and maps to provide users with detailed intelligence about the state of the business. See Figure 3 that shows $\mathrm{BI}$ and its components such as:

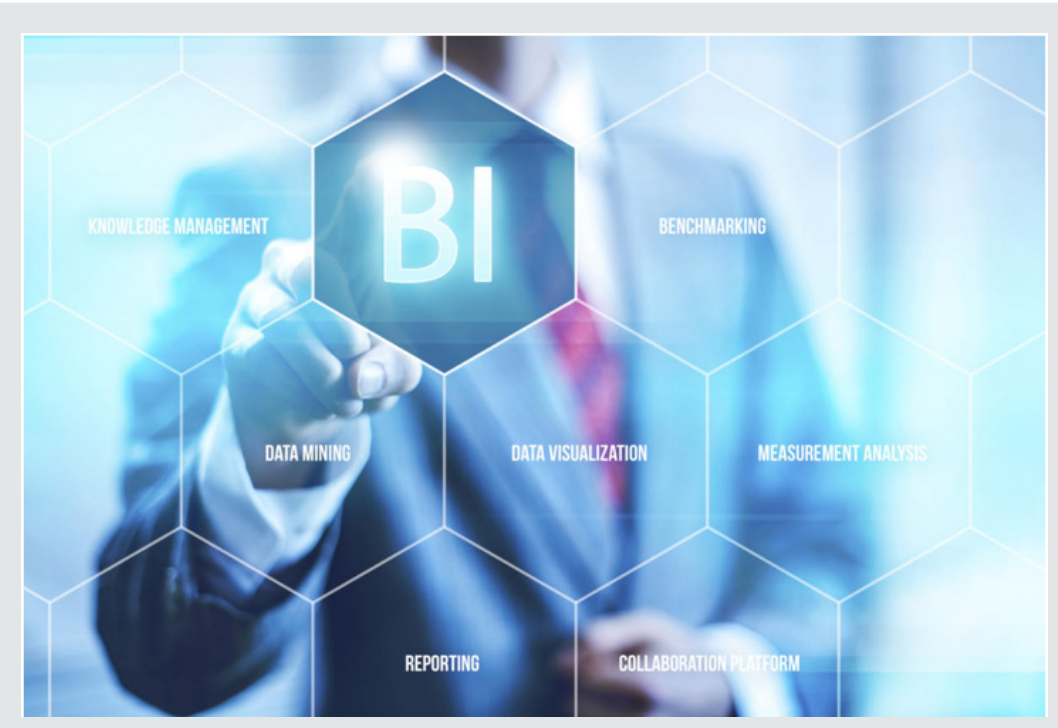

Figure 3: Business Intelligence Components. 
a. Knowledge Management

b. Benchmarking

c. Data Mining

d. Data Visualization

e. Measurement Analysis

f. Reporting, and

g. Collaboration Platform

The term business intelligence often also refers to a range of tools that provide quick, easy-to-digest access to insights about an organization's current state, based on available data.

\section{What is Artificial Intelligence (AI)}

While the main goals of Business Intelligence (BI) is aiming to streamline the process of collecting, reporting and analyzing data, Artificial Intelligence (AI) has a different approach to this goal. Using BI allows companies to improve the quality of the data they collect and the consistency with which they collect it. Thus, we need to know what the AI and its functionality and capabilities are and how it deals with data that is dumped on us with an overwhelming sheer volume and we need to have a better information in order to increase our knowledge thus having the power of a trusted decision making for our daily routine operations within our enterprise as stakeholders. Modeling human intelligence is one of the primary goals of artificial intelligence. By modeling human behaviors and thought-processes, AI programs can learn and make rational decisions. The technology professionals who build and operate AI programs are often trying to answer certain questions:
- $\quad$ Can machines learn and adapt?

- Can machines develop reliable intuition?

Exploring these questions can yield significant benefits for businesses willing to invest and experiment. Using AI-driven applications, like chatbots in early days of AI technology, can drive greater efficiency and profits. Beyond simply clarifying a messy picture, AI can provide human operators with prescriptions, and can act on those prescriptions autonomously. Unlike BI, which makes analyzing data much easier but leaves decision-making in the hands of humans, AI can enable computers to make business decisions themselves. For example, chatbots can, without human intervention, answer customer questions. Beyond simply clarifying a messy picture, AI can provide human operators with prescriptions and can act on those prescriptions autonomously. Considering what we have said about Artificial Intelligence (AI), the question now is what is AI? and how it works in a simple term. Artificial intelligence (AI) is the ability of a computer program or a machine to think and learn similar to the way that human does naturally. It is also a field of study which tries to make computers "smart ". However, there are key factors to know about AI and they are:

- It is very important to distinguish different types of Artificial Intelligence and different phases of the evolution of AI when it comes to developing application programs.

- Without recognizing the different types of $\mathrm{AI}$ and the scope of the related applications, confusion may arise, and expectations may be far from reality.

- In fact, the "broad" definition of Artificial Intelligence is "vague" and can cause a misrepresentation of the type of AI that we discuss and develop today.

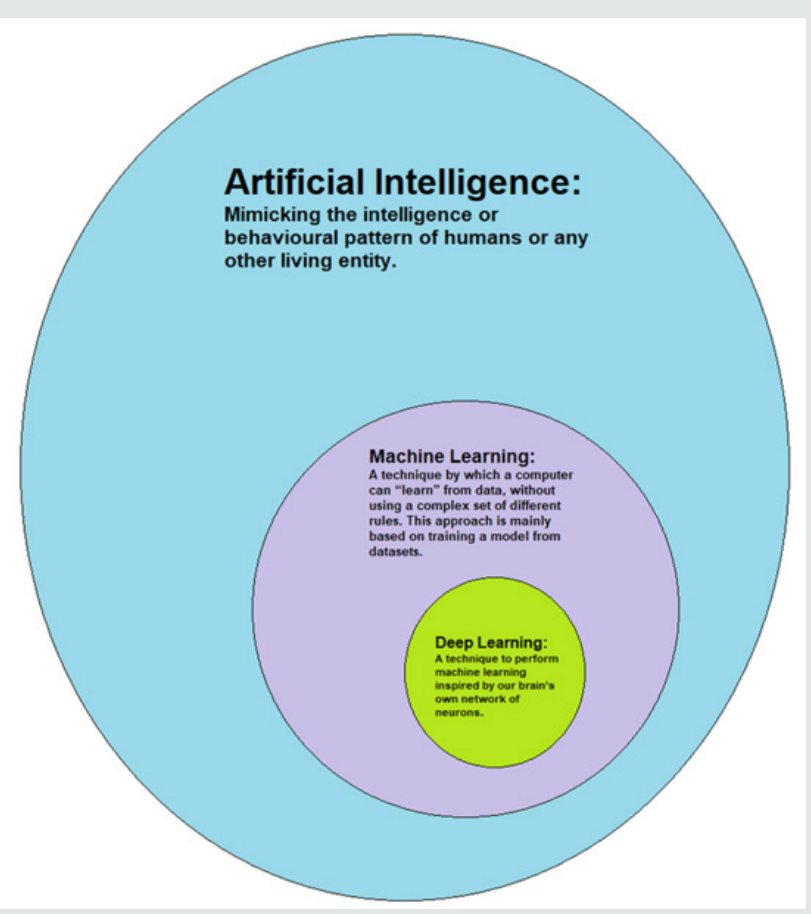

Figure 4: Presentation Artificial Intelligence in Relation with Machine Learning and Deep Learning. 
In depicted form, artificial intelligence can be illustrated in a simple for as (Figure 4). This figure is a presentation of today's supervised artificial intelligence that we are accustom to and more sophisticated version of this AI is Super Artificial Intelligence (SAI) in near future that will operate in an unsupervised mode and possibly may possess conscience capability of human being. A simple form of SAI depicted in (Figure 5) here artificial intelligence has grabbed the center stage of business intelligence, despite having been around for decades, due to the growing pervasiveness of data, the scalability of cloud computing, the availability of AI accelerators, and the sophistication of the ML and DL algorithms. Technology of AI in its today's growth, companies like IDC technology 6, predicts that by $2019,40 \%$ of Digital Transformation (DX) and big data initiatives will use AI services; by 2021, 75\% of commercial enterprise apps will use AI, over $90 \%$ of consumers will interact with customer support bots, and over $50 \%$ of new industrial robots will leverage AI. Digital transformation (DX) is reaching a macroeconomic scale. Intelligent applications based on Artificial Intelligence (AI), Machine Learning (ML), and continual Deep Learning (DL) are the next wave of technology transforming how consumers and enterprises work, learn, and play. While data is at the core of the new digital economy, it's also about how you sense the environment and manage the data from the edge to core to cloud, analyze it in near real-time, learn from it, and then act on it to affect outcomes. The Internet of Things (IoT), mobile devices, big data, AI, ML, and DL all combine to sense and collectively learn from an environment continually. What differentiates winning organizations is how they leverage that to deliver meaningful, value-added predictions and actions for improving industrial processes, healthcare, experiential engagement, or any other kind of enterprise decision making. AI business objectives are balanced between tactical and strategic, and they can range from improvement in operational efficiencies to increasing competitive differentiation, from maximizing existing product revenue to launching new digital revenue streams.

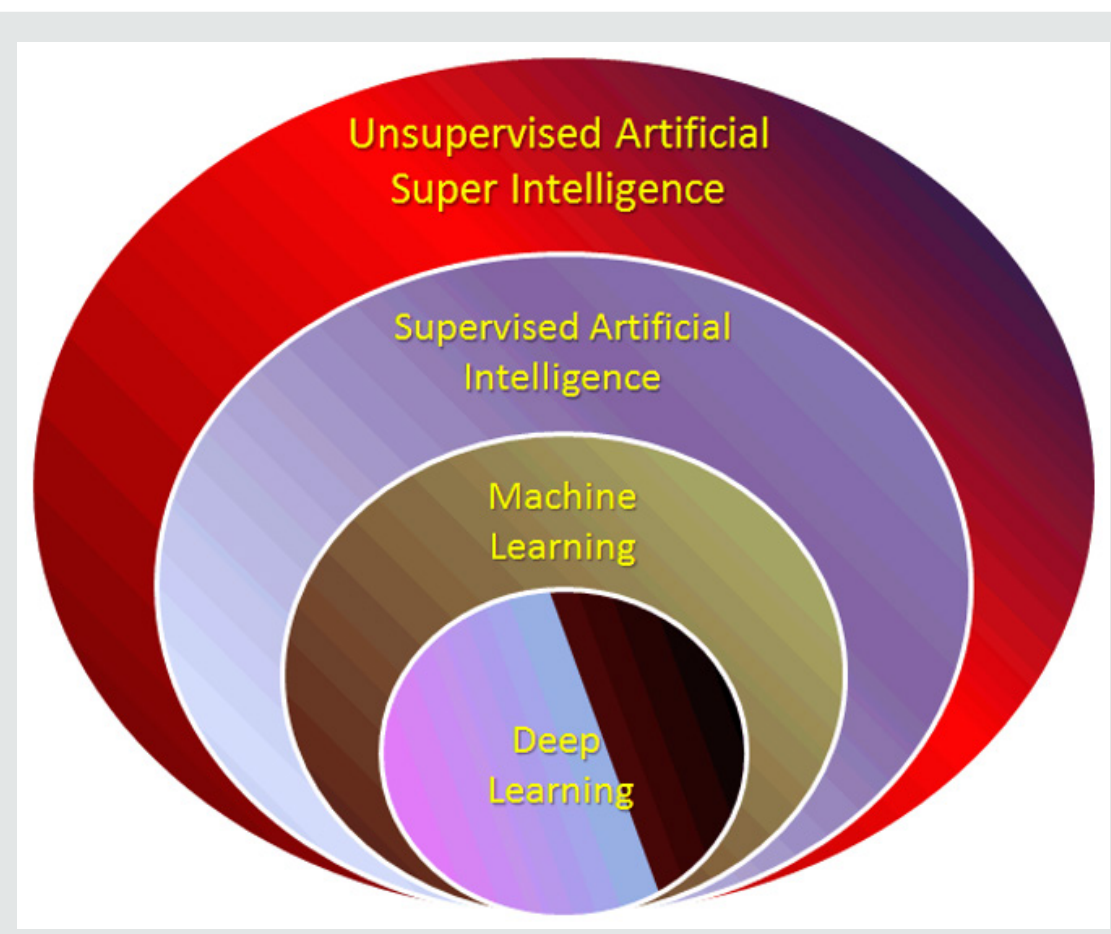

Figure 5: Schematic of A Simple Super Artificial Intelligence.

AI along with its sub-sets of Machine Learning (ML) and Deep Learning (DL), presents a collection of multiple technologies utilizing Tensor Processing Unit (TPU) and Graphics Processing Unit (GPU) (i.e., See Wikipedia or Internet for more information) 7 suggest by companies such as Google and Apple as a better and more enhanced processing units embedded within Supper Artificial Intelligences (SAIs) along with TensorFlow software interface hand-shaking with Python language written for command and control aspect of these SAIs are cable of enabling machines to sense, comprehend and act - and learn, either on their own and partner with its inventor human in order to augment human activities. Super Artificial Intelligences (SAIs) driven by Artificial Neural Network (ANN) and their capability of cognitive and natural language someday may present a future matrix augmenting its human master and even surpass him/her by being Homo-Fabians and Homo-Sapiens not too far away from the present time [1-3]. Artificial and Super Artificial Intelligence are using a capability that is known as Artificial Neural Network (ANN). It is a computational model based on the structure and functions of biological neural networks and is one of the main tools used in machine learning. They are brain-inspired systems that are intended to replicate the way that we humans learn. ANN belongs to a group of informationprocessing techniques that can be used to find knowledge, patterns, or models from a large amount of data. In general, there are three classes of Artificial Neural Networks that one needs to focus on, and they are listed below: Multilayer Perceptron (MLP).

a. Convolutional Neural Network (CNN).

b. Recurrent Neural Network (RNN). 
These three classes of neural networks provide and augment a lot of flexibility to SAIs and have proven themselves over decades to be useful and reliable in a wide range of problems that are tasked to Super Artificial intelligences (SAIs) to handle. They also have many subtypes to help specialize them to the quirks of different framing of forecasting and prediction problems and different datasets at the level of Big Data both structured and unstructured [4]. With this new technology in the horizon and rapidly growing among companies and startups in Silicon Valley in particular, one is wonder if this augmentation really helps human or hurts it by taking over of so many traditional labors works away from human by industry going toward full automation in their assembly line as an example. Compelling data reveal a discouraging truth about growth today. There has been a marked decline in the ability of traditional levers of production-capital investment and labor-to propel economic growth. Yet, the numbers tell only part of the story. Artificial Intelligence (AI) is a new factor of production and has the potential to introduce new sources of growth, changing how work is done and reinforcing the role of people to drive growth in business [5].

\section{AI Versus BI: Difference and Synergies}

Business Intelligence and artificial intelligence are increasingly crucial yet often misunderstood tools in an enterprise context. This article clarifies the differences between each field and explains how they will work together in the future. Artificial Intelligence and Business Intelligence are currently trending with add on features that provide a functional platform. Both vary on various attributes and functionalities. Artificial intelligence is based on the study and pattern of the way humans think and the algorithm mimics human brain functionality. On the other hand, business intelligence is actually technology used for making better decisions in business solutions. Comparing the Artificial Intelligence (AI) as it is today versus Business Intelligence (BI of yesterday, we can point out few points as a positive perspective for $\mathrm{AI}$, where the companies are looking for AI solutions to collect, process, analyze, and gather insights from large amounts of data that is known as Big Data (BD), helping them offer their data as a product. AI software is programmed for long hours and completes tasks faster and more efficiently. Big Data (BD) also affects organizations across every industry via certain points, such as:

\section{Contributions}

Artificial Intelligence contributes to subjects such as, government operation, education, banking, Health Care, Manufacturing, Retails and Stock Market are few that can be named as part of AI contributions. The contribution is noted widely where the algorithm follows the pattern of the human brain.

\section{Research areas}

Artificial intelligence and Business intelligence are used in research areas, but the working domains vary. Artificial intelligence is used primarily in artificial neural networks, fuzzy logic and Robotics. Artificial intelligence is known for providing suitable outputs which are used in white papers and newly published books in field of Transcranial Magnetic Stimulation (TMS), Transcranial
Electric Stimulation (TES), which are a new noninvasive of approach in the field of medicine to deal with commonly progressed depression in our today's society in particular among the youths, ages between 16 to 26 years old. Defense industries is another aspect of research area where AI is playing a big role in this industry, when it comes to Electronic Warfare (EW).

\section{Applications}

Artificial Neural Networks (ANNs) are the wave of the future, anywhere from being capable of doing everything from translating financial data into financial predictions, to mapping visual images to robotic commands, to classifying medical images for diagnostic test and so on. That why a book like this is essential reading for computer programmers, hardware and software engineers and specialists, managers, biologists, and other professionals who want to stay in the vanguard of their fields. As artificial intelligence uses algorithm that mimic the human brain, it is widely used for the application development of Gaming, Natural Language processing and Robotics. Business Intelligence is used widely for Business activity monitoring. It is mainly used as a core for reporting software applications.

\section{Missions}

Artificial intelligence is basically used to create expert systems which use human intelligence. While, the goal of business intelligence is to provide effective and efficient business solutions. Artificial Intelligence of today are foundation of future smart robots to carry on whatever tasks could be assigned to them. These robots may be designed around the idea of processing data by far faster than human brain and to be considered as plat form for computational agents of near future going forward in time. Per description by (Poole and Mackworth) [4] "A computational agent is an agent whose decisions about its actions can be explained in terms of computation. That is, the decision can be broken down into primitive operation that can be implemented in a physical device.

\section{Drawbacks}

Artificial intelligence includes issues like threat to privacy and threat to safety. Business intelligence includes drawbacks with two types: one with organization and people working with the organization and the other with technology and data. We took a look at the distinguishing points of business intelligence and artificial intelligence. It is also important to understand the difference between them from a technical perspective. Let us analyze the algorithm used by artificial intelligence and business intelligence. Artificial intelligence uses a technique that uses Breadth First Search (BFS) algorithm. Breadth first search algorithm follows a pattern to search for the neighboring nodes in tree data structure following each level involved in it. The pattern can be followed using FIFO. It provides a shortest path to the solution. Note that, Breadth-first search is an algorithm for traversing or searching tree or graph data structures. It starts at the tree root and explores all of the neighbor nodes at the present depth prior to moving on to the nodes at the next depth level (Figure 6). It uses the opposite strategy as depth-first search, which instead explores the node branch as far as possible before being forced to backtrack and expand other nodes. 


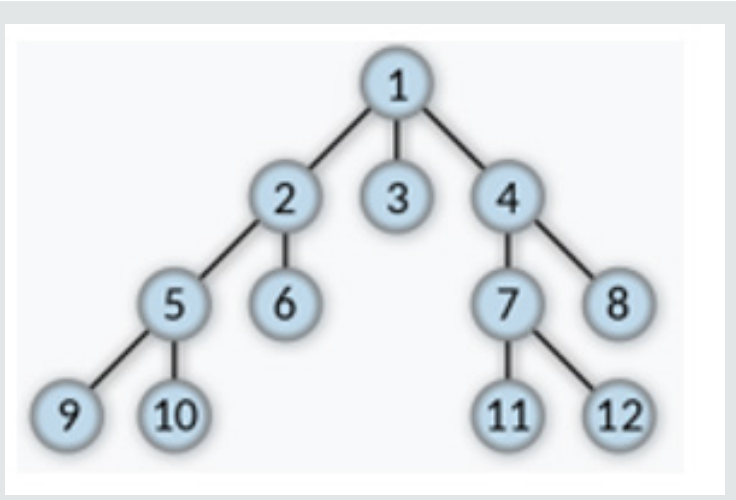

Figure 6: Order in Which the Nodes Are Expanded.

Moreover, Business intelligence extracts the predictive information in such a format which is understood in layman terms. It follows the decision tree algorithm. All the information extracted using business intelligence marks as predictive information. Business intelligence does not focus on sorting. It uses a linear aggression module for classifying the data. This approach of business intelligence is widely used for data mining process.

\section{Integration of Business Intelligence with Artificial Intelligence}

Business Intelligence (BI) and Artificial Intelligence (AI) are monitored together in the field of data science. The combination is considered as a perfect match. Consider the Venn diagram below to understand the combination of artificial intelligence and business intelligence with data science. Data science is primarily used by the research analysts to achieve output for the researchers conducted in artificial intelligence and business intelligence. The study says that artificial intelligence is coping up with many algorithms in comparison with business intelligence (Figure 7) is illustration of $\mathrm{BI}$ and $\mathrm{AI}$ combined. The combination of algorithms with Artificial intelligence, business intelligence and data science are massively used for big data management. Business intelligence with data science concepts are used for statistical analysis. Artificial intelligence with data science is used for supervised machine learning primarily. The combination can be used to create innovate dashboards for upcoming technologies. Business intelligence with artificial intelligence tools like artificial neural networks is primarily been used in meteorological departments and the study of earthquakes (seismology).

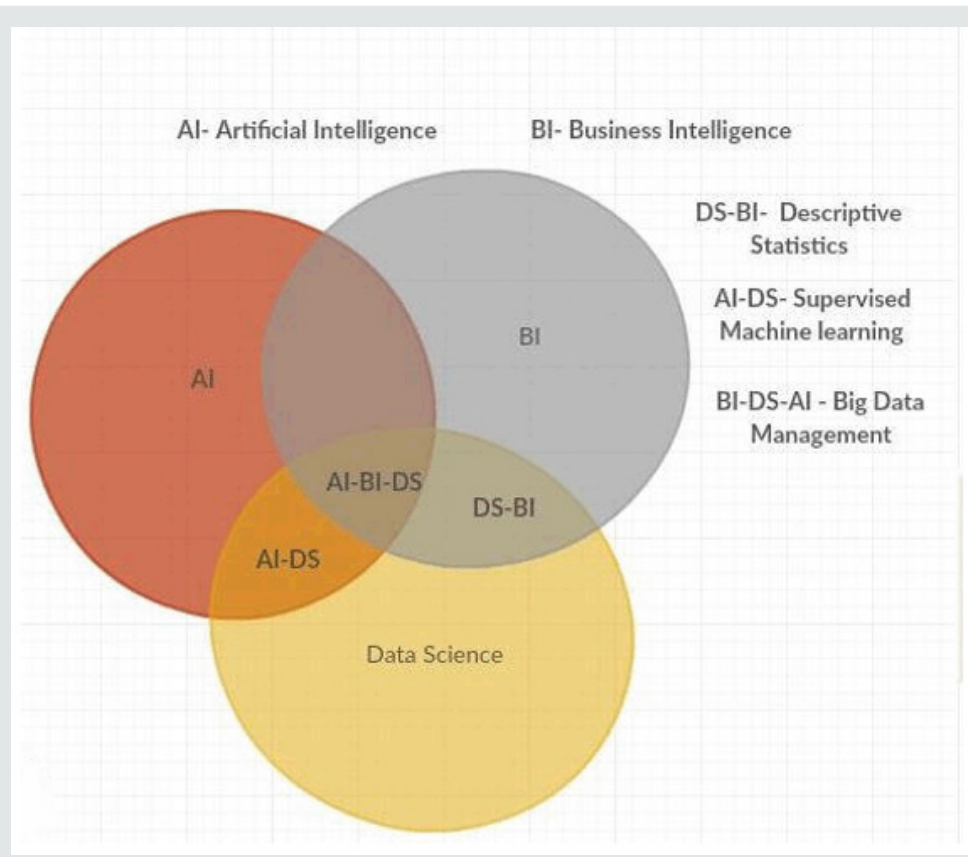

Figure 7: Business Intelligence and Artificial Intelligence Combined.

\section{Artificial Intelligence is the Future of Business In-} telligence

From the time that us as developer of Business Intelligence (BI) start implementing it into our day-to-day operation as a tool, augmentation of Artificial Intelligence (AI) has been top priority. Considering that volume of data both structured and unstructured growing to the level of Big Data and continue growing beyond need for a new tool such as AI and its components Machine Learning (ML) and Deep Learning (DL) is becoming a necessity of our daily business operations. Given our mandatory encountering the Internet of Things (IoT) to make our fast paste operation of our enterprise based on demand leaves us no choice except looking into Artificial Intelligence at present and sooner but not later start exploring the Super Artificial Intelligence (SAI), where we need dealing with an unsupervised AI. It is very clear, and it has been for some time that AI will play a big part in the future of business intelligence. For example, some of the recently developed AI capabilities seem to come straight out of a science fiction movie: voice recognition and digital assistants, task automation, medical diagnoses, and facial recognition systems-and systems to fight those facial recognition systems. Not only is AI capable of doing all those fantastic science-fiction things or Intent of Things (IoT), but it is 
also capable of making a big difference in our analytics world. Thus, Artificial Intelligence (AI) in business intelligence is influencing the way Business Intelligence used to work and helps democratize data and improve analytics adoption.

Radical advances in computing power, in data analytics, thus predictive analytics, machine learning, deep learning and AI have opened the door to a new generation of Business intelligence (BI) tools. If implemented properly, AI pulls actionable insights from complex data sets and automatically recommending next actions. AI provides data points exactly where and when they are needed most with very little work required by the user, given its sub-function of Deep Learning (DL), where it passes the right information to Artificial Intelligence (AI) via Machine Learning
(ML) by comparing historical data with incoming present data and learn from such comparison to be able to forecast the future events. Such capabilities will make the enterprise to be as resilience as possible, using techniques such as Business Resilience System (BRS) [5-7]. AI capabilities and functionalities bridges the gap for business users who do not have deep technical knowledge, yet they are end users and operators of organizations and enterprises. Because AI can analyze massive quantities of data and deliver recommendations based on that data, it makes analytics and big data insights accessible and understandable to the average usernot just data scientists. Where a sharp divide once existed between two groups of users-those familiar enough with data science to glean value from the data themselves, and those who are not-AI bridges that gap and delivers insights in a digestible format.

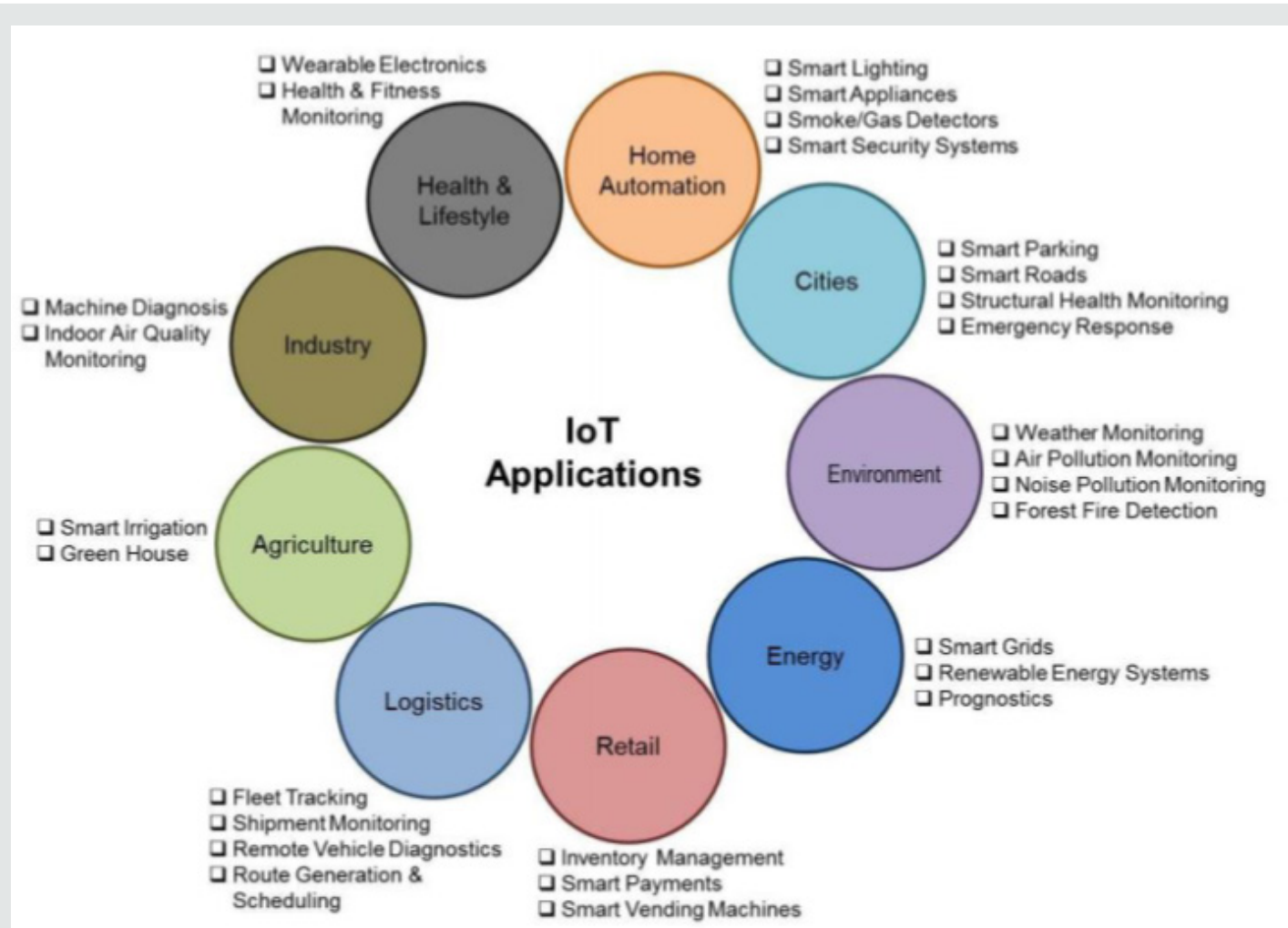

Figure 8: Business Segments for IoT/IIoT Implementation (Courtesy of What is next for IoT and IIoT, Australia 2015 by Ahmed Banafa).

AI is already getting better at identifying trends and extracting insights from complex data than humans are, and these abilities will only improve over time. Rather than continuing to pour money into tools that require employees to spend their time manually analyzing data and making mundane decisions, business organizations should invest in the future of BI and ai-powered systems that automate the bulk of these processes and allow their talent to focus on the strategic problems that really move the needle [8]. The $21^{\text {st }}$ Century has opened up as well as recognized another dimension to our industry of past and present as renaissance of industrial revolution, where the Information and Communication Technology (ICT) plays as a driving factor of Internet of Things (IoT). This industrial revolution will bring big changes in all business segments, enormously impacting people's lives as it is presented by (Figure 8) here. Companies within today's industrial environment from ICT business segments in this new market, according to some approximations, will be worth over \$152 Billion Dollars by year 2022 per I-Scoop 2016 prediction. They will have crucial roles in this new industrial period in changing and adjusting their revenue-earning projections accompanied by investment in relevant resources. instance, mobile telecom operators will have to reorganize and reposition themselves to become ICT operators, and companies in all business segments will have to do the same in changing and adjusting their business activities to include their organization, products and services.

Internet of Things (IoT); Industrial Internet of Things (IIoT); Over the Top (OTT) Applications; Artificial Intelligence (AI); Cloud Services (CS) and many other items are considered as part 
of ICT going forward. These changes for example will bring new approaches for creating smarter telecommunication products and services in mobile industry and consequently, customers will able to use completely new types and kinds of products and services in telecommunication markets as simple aspect of IoT along with growth of data to the level of Big Data and implementation of Supper Artificial Intelligence (SAI) as a processing tools by filtering these data in a right direction, Information and communication technologies have had a profound effect in lots of marketing as a tool $[8,9]$. Although artificial neural networks have simpler processing units than typical Central Processing Units (CPUs), their unique form of parallel processing and tremendous number of interconnections make them incredibly versatile problem solvers. This far-reaching source gets you in on the ground floor of this state-of-art technology, giving you a complete overview of neural network capabilities that drives artificial intelligence, limitations, components, and applications in diverse fields [8,9]. An important aspect of this approach by us is to exam biological neural systems and how, artificial neural network is based on them, and, driven by them as well. Key areas discussed include structural diversity... temporal...origins of artificial neural systems...brain structure and function...biological nerve cells... synapses...what is random and what is fixed in the brain's neural networks...and how biological systems really compare to computational neural networks [3].

For us to deliver such momentum of knowledge in Neural Networking to our reader, we have reached to experts in the field and with their permission we have quoted their notes, lectures and their presentations from their web site at various universities and industries. Our many thanks go to experts like Professor Ingrid Russell of the University of Hartford and permission from Collegiate Microcomputer, Lectures by Kiyoshi Kawaguchi and Professor David Beeman of University of Colorado, Department of Electrical and Computer Engineering on Computational Neuroscience. In addition, some information that are used was permitted by Dr. David Leech Anderson, Professor of Philosophy at Illinois State University and art works are provided by, "graphic artists at The Mind Project" [10].

\section{Conclusion}

Artificial intelligence is the core for applications and enterprises which use human intelligence for basic functionality. The human intelligence can be used as a symbol structure and symbolic operations which are primarily used in digital computers. Business intelligence focuses on report management and analyzing data with data mining and data warehousing procedures. The organization uses business intelligence to gain achievable insight of the data. This includes basic operational analysis or spreadsheet analysis. It is mandatory to analyze the requirements and select the suitable module for application development using artificial intelligence or business intelligence or a combination of both. In summary, Artificial Intelligence enables us to put our data in a perspective that will work for us rather than overwhelming us with its sheer volume. By analyzing all the data that has been collected and delivering real time recommendations, AI enables you to actually use your data to your competitive advantage. If your competitors aren't using AI, they'll be stuck sifting through mountains of data on their own. By using AI, you'll be able to harness the power of the data you collect identify more trends, build recommended actions based on those trends, and ensure that those actions and surrounding context are delivered to your end users exactly when and where they need them. While AI, like any technology, certainly has some limitationsmost notably when it comes to cybersecurity or inherent bias-the potential benefits are enormous. In spite of this, many companies are lagging behind when it comes to recognizing $\mathrm{AI}$ as a trend in the future of business analytics and intelligence. Those companies that begin to implement AI will soon find themselves outperforming their competitors when it comes to improved overall business performance and higher revenue.

As final steps of this conclusion, both AI and BI have key, and in some cases overlapping, enterprise applications. Put it in a simple statement artificial intelligence (AI) explores the use of computer systems to mimic various attributes of human intelligence, such as problem solving, learning, and judgment. Though in its technological infancy, businesses see huge potential in AI for speech recognition, decision-making, and everything in between. While, Business Intelligence (BI) refers to the use of various technologies and tools to collect and analyze business data. The main purpose of $\mathrm{BI}$ is to provide companies with useful information and analysis to aid decision-making. Using BI allows businesses to make decisions nearly five times faster than they otherwise could. On the other hand, Artificial Intelligence puts BI in different perspective, knowing that, Artificial Intelligence (AI) is the ability of a computer program or a machine to think and learn similar to the way that human does naturally. It is also a field of study which tries to make computers "smart".

\section{References}

1. Leonardo dos Santos Pinheiro, Mark Dras (2017) Stock Market Prediction with Deep Learning: A Character-based Neural Language Model for Event-based Trading. P: 6-15.

2. https://www.sas.com/en_us/insights/analytics/machine-learning.html

3. Boris B (2019) Using the latest advancements in deep learning to predict stock price movements. Towards data Science.

4. David LP, Alan KM (2017) Artificial Intelligence, Foundations of Computational Agents. $2^{\text {nd }}$ Cambridge University Press, USA.

5. Zohuri B, Mossavar Rahmani F (2019) Artificial Intelligence Driven Resiliency with Machine Learning and Deep Learning Components. International Journal of Nanotechnology \& Nanomedicine 4(2): 1-8.

6. Anthony L, Walden (2007) Understanding Data, Information, Knowledge and Their Inter-Relationships. Journal of Knowledge Management Practice 8(2).

7. Bahman Zohuri, Farhang MR (2020) A Model to Forecast Future Paradigms: Volume 1: Introduction to Knowledge Is Power in Four Dimensions. Apple Academic Press pp: 464.

8. Zohuri B, Moghaddam M (2017) Business Resilience System (BRS): Driven Through Boolean, Fuzzy Logics and Cloud Computation: Real and Near Real Time Analysis and Decision-Making System. $1^{\text {st }}$ (edn.), Springer Publishing Company, USA.

9. Zohuri B, Moghaddam M (2019) Artificial Intelligence Driven Resiliency with Machine Learning and Deep Learning Components. Journal of Communication and Computer 1-13.

10. Roman Stanek (2018) Artificial Intelligence is the Future of Business Intelligence. 
(C) (P) This work is licensed under Creative To Submit Your Article Click Here: Submit Article

DOI: $10.32474 /$ MAMS.2020.02.000137

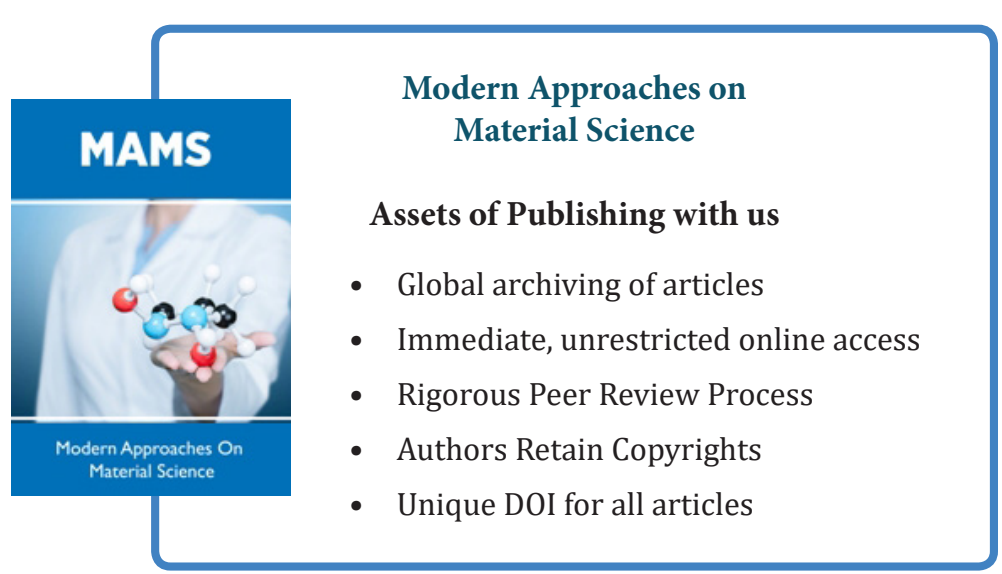

